\title{
Wireless Body Area Network and Sensor Data Interoperability
}

\author{
Joy Alatta \\ Walden University,joy.alatta@waldenu.edu; joy.alatta@learnatek.com
}

\begin{abstract}
The monitoring of maternal and child health, using wearable devices made with wireless sensor technologies, is expected to reduce maternal and child death rates. Wireless sensor technologies have been used in wireless sensor networks to enable the acquisition of data for monitoring machines, smart cities, transportation, asset tracking, and tracking of human activity. Applications based on wireless body area network (WBAN) have been used in healthcare for measuring and monitoring of patient health and activity through integration with wearable devices. Wireless sensors used in WBAN can be cost-effective, enable remote availability, and can be integrated with electronic health record (EHR) management systems. Interoperability of WBAN sensor data with other linked data has the potential to improve health for all, including maternal and child health through the improvement of data access, data quality and healthcare access. This paper presents a survey of the state-of-the-art techniques for managing WBAN sensor data interoperability. The findings in this study will provide reliable support to enable policymakers and health care providers to take action to enhance the use of e-health to improve maternal-child health and reduce the mortality rates of women and children.
\end{abstract}

Keywords: Sensor data, wireless body area network, wearable devices, sensor data interoperability.

\section{Introduction}

Expectant mothers that live in areas where there is a limited medical facility, belong to the lowincome group, and have to work to make a daily living may not always attend clinics for a regular checkup. Say et al. (2014) stated that an estimated 287000 maternal deaths in 2010 were mostly from low-income and middle-income countries. According to the World health organization (WHO, 2019), $94 \%$ of all maternal deaths occur in low and lower-middle-income countries. Having a regular checkup can enable health care providers to detect and prevent pregnancy-related problems and lower the risk of potential pregnancy complications (Manda-Taylor, Sealy and Roberts, 2017). Expectant mothers who cannot attend regular checkups miss out on routine checks for vital signs such as blood pressure, temperature, heartbeat rates and others. The hectic pace of the modern lifestyle makes it difficult for women to meet the recommendation of 150 minutes of moderate to vigorous physical activity in a week (Reed et al., 2015). e-Health can enable and empower programs and strategies for monitoring women's cardiometabolic health. Roy, Srivastava and Gururajan (2018) identified that lifestyle diseases are a major threat to human life and low health literacy has a relationship to poor health behaviors and management of chronic diseases. Other implications of low health literacy are delayed diagnoses, poor adherence to treatment regimens, increased morbidity and mortality (Rahmani and Brekke, 2013; Kim and Xie, 2015).

Wearable devices have been used in many instances for continuous monitoring of vital signs (Petit and Cambon, 2016) and improvement in the quality of care (Mony et al., 2018). The use of wearable devices is not designed to replace medical practitioners, but rather to assist medical practitioners to evaluate the patient even when the patient is physically absent. Using wearable devices for remote monitoring of patient health can also reduce healthcare costs (Prabhakaran, Ajay and Tandon, 2019). Wearable devices are being used to collect data for diagnosis to alleviate the dangers of maternal and 


\section{Joy Alatta / Wireless Body Area Network and Sensor Data Interoperability}

child death rates. The challenges of maternal child health $(\mathrm{MCH})$ could be addressed by using mobile and wearable devices. The use of mobile and wearable devices is likely to encourage the usage of mobile health resources (Gay and Leijdekkers, 2015), improve awareness of potential health issues and increase the speed of delivery of service.

Wireless sensor technologies have been used actively for measuring and monitoring of patient's health and activities using wearable devices (Nebeker, Linares-Orozco and Crist, 2015). Wearable devices can play dynamic roles in maternal-child health care when they are used effectively, and the data from the sensors are formatted using standard semantics and ontology. Sensors connected to wearable devices allow for the collection of data helps to improve patient's awareness of health challenges, enables patients to participate in the collection of quality data, and increase affinity to use healthcare facilities. However, user involvement in the design of e-health is likely to be a core factor in the adoption of e-health by pregnant women. The increase in the use of smartphones and devices (Azevedo et al., 2015; Petit and Cambon, 2016) is an indication that smartphones could be successfully used by potential users and supports the application for health interventions.

Several studies conducted in researching and developing wearable and implantable devices (Lee, 2016), indicates that there are advancements in the use of wireless technologies are increasing. ehealth technology can be used in health intervention strategies (Amoakoh-Coleman et al., 2016). Kaewkungwal et al. (2010) discussed the better border project, an application designed to manage and monitor antenatal care (ANC) and expanded immunization services (EPI). It was concluded that there was an improvement in readiness to access health care services and improvement in the time taken to deliver health care to patients. de l'Aulnoit et al. (2018) developed a mobile data module for fetal monitoring, which sends data to a hospital information system that hosts electronic medical records (EMR). Mony et al. (2018) designed, developed and tested a wearable sensor device for remote biomonitoring of body temperatures in mothers and newborns in southern India as a proof of concept. The devices which were secured on to adults and neonates transmitted the data via the gateway device for secure storage and retrieval for analysis. The device was described as promising in terms of feasibility, safety and accuracy. The heterogeneity of wireless devices leads to the production of heterogeneous sensor data that lives in silos, which ultimately limits its use by clinicians (Reda, Piccinini and Carbonaro, 2018).

\section{Wireless Body Area Network}

The WBAN enables real-time monitoring of sensors used in the healthcare, military, sports, entertainment and can ensure quality healthcare (Shokeen and Parkash, 2019). WBAN sensors are used in the construction of devices that may be positioned in certain parts of the body. The devices constructed with WBAN may be described as wearable devices or implantable devices (Sudjai et al., 2016). The sensors on the devices work to provide continuous monitoring and sensing of physiological changes (Compton et al., 2012). The sensors acquire data that is consequently transmitted to an information system connected to the device (Lo and Yang, 2016). As a real-time network, the accuracy of WBAN sensor data may be challenged by environmental constraints, fault tolerance and security (Shokeen and Parkash, 2019). Wearable devices are used to monitor heart rate, body temperature, blood glucose level and others (Lee, 2016). The sensor types used in WBAN include ECG sensor, motion sensor, heart rate sensor, pressure sensor and positioning sensor (Kaur and Malhotra, 2017). WBAN sensors can be used in the medical and non-medical enabled devices 
(Kim and Kim, 2016), enables improvement in communication with patients, and in the early detection and prevention of diseases. Quality of life of patients (Mony et al., 2018) is improved where patients do not have to be confined in a bed for the purpose of monitoring.

WBAN sensor data acquire a variety of data (Yilmaz, Foster and Hao, 2010) types of which some may be delay-sensitive or reliability sensitive depending on the part of the body the data is acquired. Data from ECG and EEG may need to be transmitted continuously and is highly data-centric. The diverse nature of the data may mean different parameters; computation method, storage and energy requirements (Peng, Goswami and Bai, 2018). Wearable devices are used in varied conditions, which subjects the devices to fluctuating and unreliable networks, and may result in data inconsistency. The success of using smartphones and other wearable devices to monitor health in real-time depends on real-time data exchange. The quality of the data that WBAN sensors send out is what enables remote patient monitoring. There is a need to understand how data collected from wearable devices can be used to deliver health solutions to users (Rhayem, Mhiri and Gargouri, 2017).

\section{Interoperability of data}

Interoperability attempts to transform low-level sensor data to high-level knowledge that is human and machine-readable (Honti and Abonyi, 2019). Hospitals may have healthcare applications in different databases or patients may use different healthcare facilities for other health issues. These applications may be designed with different models. Interoperability enables access to data from disparate sources (Mavrogiorgou et al., 2019). Integration of sensor data relies on the combination of data from devices manufactured by different vendors (Jabbar et al., 2017). Interoperability enables different types of information and communication systems to have an effective, consistent and accurate exchange of data that can be interpreted by all the communicating systems (Iroju et al., 2013). Many integration standards have been used in health data integration including HL7 standards, Digital Imaging and Communications in Medicine (DICOM), Clinical Information Modeling Initiative (CIMI), clinical coding systems (SNOMED-CT, ICD, Logical Observation Identifiers Names and Codes (Peng et al., 2018). Interoperability has been addressed from different perspectives using different standards (Kumar et al., 2016).

Peng et al. (2018) noted the use of health data interoperability standards including HL7 Fast Healthcare Interoperability Resources (FHIR) and IoT ontology including Semantic Sensor Network (SSN) in the interoperability of sensor data. Wearable and implantable devices such as ECG, EEG, hearing and cochlear implant, glucose monitoring may be described as a low-end or high-end device due to the level of computational capability, processing power and communication capacities. Various WBAN related devices have different processor speed, random access memory (RAM), communication and power requirements. WBAN sensors are made by multiple vendors and rely on diverse wireless and networking topologies (Vengadeswaran et al., 2019). The wearable devices constructed with these devices are expected to communicate seamlessly to exchange messages. While trying to exchange messages, the network handles issues of addressing, routing, resource optimization, security and quality of service (QoS). There are different types of interoperability namely; structural interoperability, syntactic interoperability, and semantic interoperability. 
Joy Alatta / Wireless Body Area Network and Sensor Data Interoperability

\section{Structural interoperability}

In structural interoperability, a midlevel type of interoperability is defined for data information exchange (Studeny and Coustasse, 2014) where the systems read and understand the information at a granular data level. Structural interoperability describes different ways of storing data using predefined formats for the data exchange so that the received data can be interpretable (Zhang et al., 2018).

\section{Syntactical Interoperability}

This refers to the data format schema and interfaces required for the exchange of information between different systems using compatible interface and compatible data formats (Garde et al., 2007). The heterogeneous nature of WBAN sensors creates challenges in encoding and decoding rules defined to enable the exchange of messages for each resource. Schema differences such as WSDL, Rest API and format differences such as XML or JSON contributes to the challenges. Syntactic interoperability provides the foundation for formatting data through common data exchange format and common protocol for structuring data (Iroju et al., 2013). Metadata repositories support syntactical interoperability through compatible data formats (Vengadeswaran et al., 2019). In syntactic interoperability, syntax can be considered as the grammar to convey semantics and structure (Garde et al., 2007). Syntactic interoperability provides no guarantee that the data received can be interpreted or processed by the receiving system.

\section{Sematic Interoperability}

Semantic interoperability relates to different ways of interpreting the meaning of data and the ability of computer systems to communicate and exchange data using a shared vocabulary and meaning (Jabbar et al., 2017; Zhang et al., 2018). The communication uses a simultaneous transmission and includes the metadata, with each data element linked to a shared vocabulary, and each transmission of data including a self-describing universal information package. As a universal information package, it is independent of information systems. The shared vocabulary in the information package is linked to an ontology that enables machine interpretation (Garde et al., 2007), inferences and logic. The ontology provides a basis for users to be able to interpret existing terms and also be able to create new terms in a way that other systems can interpret the term. Ontologies support semantic interoperability through the provision of sets of concepts with unambiguous meaning. The concept of semantic web (WC3) described the standards for the provision of web-scale data exchange, including federation and inferencing. The ontology keeps the entities relationship, the entities, rules and axioms for understanding data elements. The heterogeneous nature of devices in a WBAN system requires the use of semantic descriptions to enable interoperability. Semantic interoperability provides the highestlevel type of interoperability by using data exchange standards and data coding for shared interpretation (Studeny and Coustasse, 2014). Using a shared meaning in semantic interoperability enables all the communicating systems to be able to able to homogeneously process information from heterogeneous devices (Iroju et al., 2013). This paper has focused on semantic interoperability.

\section{Related Works}

Gay and Leijdekkers (2015) have been working on a project that maintains their mobile application called myFitnessCompanion, which integrates health data from diverse operating systems such as 
Android, Apple IOS and Microsoft mobile. The sensor data are from commercial off the shelf trackers such as Fitbit, iHealth, jawbone, Withings etc. The application can support up to seven active sensors to accommodate users with multiple monitors, and can tag the source of each data. The project used the HL7 standards to achieve foundational, structural and semantic interoperability. The application formats the data in JSON and XML. Kasthurirathne et al., 2015) leveraged on the open medical record system (OpenMRS) to integrate data with the FHIR, which resulted to the development of the FHIR API. The FHIR maintained by the HL7 (Bender and Sartipi, 2013; Cornet, 2015) enables the connection of different data elements using XML based standards to specify the structure and semantics of the patient's data. The FHIR is expected to improve the interoperability of data to overcome the shortcomings of other previous standards (Saripalle, 2019).

Huang and $\mathrm{Wu}$ (2016) focused on the tasking capability of the internet of things and proposed an interoperable solution called tasking capability description which allows users to control different IoT devices using a uniform web service interface. The work is based on the OGC SensorThings API and produced Extended SensorThings API. The Extended SensorThings API proposed automatic translation between device protocol through the use of keyword replacement. With this solution, devices can use the defined data model to describe their tasking capabilities. Ivaşcu, Frîncu, and Negru (2016) built a semantic data model using domain-specific ontologies for data description by converting the data to RDF/XML. A multi-agent architecture was used for the data stream and data stream processing that could manage a large amount of sensor data. Hussain and $\mathrm{Wu}$ (2017) presented a model to manage the heterogeneity in data syntax and semantics. The model used two types of semantic validation in data integration through RESTful web service of SensorThings API, and validation of the semantics used in the model and JSON documents.

Peng et al., (2018) applied semantic web technologies to integrate the health data and home environment data from heterogeneously built services and devices using web ontology language (OWL)-based integration ontology. The application modeled health data from HL7 FHIR standard implemented services, normal Web services and Web of Things (WoT) services and Linked Data together with home environment data from formal ontology-described WoT services. The outcome is an integrated machine-readable data, annotated with semantics and ontological links for inter-system usage. Vengadeswaran et al. (2019) used semantic annotation through standardized terminologies and classifications to foster semantic interoperability. The work extended Samply.MDR to store semantic information. Successful enrichment of data elements with UMLS codes was shown by interconnecting Samply.MDR and MDM-Portal.

W-IoT Plug and Play project is an attempt to achieve an open and interoperable sensor web and internet of things end-to-end architecture based on the OGC SensorThings API. Huang and Chen (2019) applied the data model in the Extended SensorThings API in Huang and Wu (2016) and used it as a service layer to support tasking and sensing capabilities. The proposed sensor web and internet of things plug and play can automatically register embedded devices. The model worked by enabling embedded devices to be self-describing. To be self-describing means that the devices introduce their metadata, sensing and tasking capabilities, and device communication protocols. 


\section{Joy Alatta / Wireless Body Area Network and Sensor Data Interoperability}

\section{The need for interoperable data}

The proliferation in the use of wearable devices in healthcare necessitated the demand for understanding how to foster interoperability among heterogeneous devices and the data each device produces (Haller et al., 2019). There is a need to properly access the values across devices and information systems. Health data in legacy systems live in silo locations (Gay and Leijdekkers, 2015), coded with diverse data formats and cannot be easily accessed for use in rich analytics (Dimitrov, 2016). Aggregated data is required to achieve the objective of WBAN due to the ability to reduce overhead and enhances transmission efficiency. WBAN data is likely to make more sense after normalization through consistent format and shared semantic to enable data sharing, effective use of machine learning and artificial intelligence (AI) technology (Kim et al., 2016). Interoperable data facilitates clinical decisions by enabling clinicians to identify patterns and make predictions based on the identified patterns and trends. The realization of the objective of mobile healthcare depends on the ability to gather, process, store and share the sensor data (Choi et al., 2015). The use of patientgenerated health data for the provision of precision medicine emphasizes the coordination and integration of care using appropriate technologies to connect the patient and the healthcare system.

\section{Semantic Web technologies}

Semantics is a combination of ontologies, contexts and structured metadata. The semantic web is used for integrating diverse web data to enable easy search, reuse, and linking of data. Semantic web technologies act as integrators of various web services, databases and other formats for the presentation of data using ontologies and data annotation (Ahmed and Ahmed, 2018). Semantic technologies are used for managing, querying and integrating sensor data (Compton et al., 2012). The issues of data interoperability have existed as long as there were several types of information systems. The interoperability of information systems has become relevant in a web 2.0 world where data of varied formats and types are shared and received. Wearable devices are constructed with different types of sensors and actuators manufactured by different vendors and the various systems that store the data need to be able to communicate and exchange information (Puustjärvi and Puustjärvi, 2015). Each vendor seems to have different data formats, system architecture, networking standards and services (Seydoux et al., 2016). The heterogeneity of the sensors, architectures and semantics creates diverse interoperability issues which can be described as device interoperability, networking interoperability, systematic interoperability, semantic interoperability and platform interoperability (Noura, Atiquzzaman and Gaedke, 2018).

A major goal of the semantic web is to ensure machine-interpretable data at the web-scale and has proven to be effective in mitigating heterogeneity through semantic interoperability for easy integration of data application (Seydoux et al., 2016). Semantic interoperability enables the extraction of new knowledge that can be used to build smart solutions and interoperability among various data processes (Reda et al., 2018). The use of shared semantic meanings enables integration of data from heterogeneous sources (Puustjärvi and Puustjärvi, 2015) because the definitions attached to the meanings provide a foundation for provenance and reasoning (Compton et al., 2012). Semantic-based knowledge representations (He et al., 2017) contributes to surmounting the challenges of innovative semantic-based solutions to heterogeneous data. 


\section{Semantic Ontology}

Semantic ontology is an approach to managing semantic interoperability by expressing the concepts in a domain, including its attributes and interrelationship using a formal logic-based specification (Kumar, Kumar and Raghuveer, 2017). The same formal logic specification is then applied to similar data from other sources. Ontologies can describe and enrich knowledge within a relevant context (Kaur and Aggarwal, 2017). Domain ontology presents a formal description of knowledge within a specific domain such as the healthcare domain. A domain ontology in healthcare will define knowledge representation within the domain. Ontologies provide the foundation to link one information to the other, ensures better data management, interconnectedness and interoperability (Maarala, Su and Riekki, 2016). The major challenge of interoperability is to ensure that diverse products, processes and information systems can connect and exchange information.

The collaboration and interconnection of diverse sensor data can promote contextual information exchange and sharing, and enable the extraction of new types of knowledge (Honti and Abonyi, 2019). Ontologies used in the internet of things which also include WBAN sensors can be described as device ontology, domain ontology and estimation ontology (Hachem, Teixeira and Issarny, 2011). An ontology is composed of classes, relations, attributes and individuals where classes are the concepts that require description. The sub-classes are the relations, the attributes describe the properties and characteristics of the concept. Individuals may be instances of classes or their properties.

WBAN applications need to automatically process and exchange information with other applications, thus creating the need for appropriately and formally defined syntax and semantics. The W3C web ontology language (OWL) has been a standard for representing ontologies. Other ontologies are SOSA/SSN (Janowicz et al., 2019), and COBRA-ONT (Bajaj et al., 2017). The semantic ontology approach introduces flexibility in data integration, maximizes different granular of data and copes with temporal changes in data definition (Liyanage, Krause and de Lusignan, 2015). An understanding of the meaning of a piece of data is crucial to solving the problem of heterogeneity (Jirkovský, Obitko and Mařík, 2016) in sensor data. With a standard definition of the contexts and meaning, metadata and provenance can be improved. Though ontologies enhance data interconnection and interoperability, it could also delimit that objective when the available property constraints are limited.

\section{Semantic Contexts}

Contexts are described from the standpoint of conveyance of meanings. This could include body language and vocal. Contexts in semantic web technologies include both the individual meaning of the word and the domain meaning of the word. Semantic contexts use natural language concepts to represent words and phrases within a specific domain by using the logical structure in the word or phrase to identify and understand the relationship between classes. Understanding the relationship between classes enables the linking of multiple descriptions for a class (Beek, Schlobach and van Harmelen, 2016). Maintaining links and relationships between entities in heterogenous datasets is crucial to semantic web technology (Idrissou et al., 2017). 
Joy Alatta / Wireless Body Area Network and Sensor Data Interoperability

\section{Semantic Metadata}

Metadata is the description or data about a piece of data. It is used to apply data descriptions in a standardized way. Using a standard description removes ambiguity in a given context. Metadata represents the properties or values specified for a piece of data. The description may be driven by an ontology for the domain. Semantic metadata improves document search, linking and integration. The resource description framework (RDF) is an example of a metadata model (Maarala et al., 2017) and uses various syntax notations and data serialization formats to model data. Other standards used in the semantic web are the SPARQL Protocol and RDF Query Language (SPARQL) and OWL (AltarSamuel, Costin and Enache, 2015). Semantic metadata enables the description of a web resource. RDF is constructed with web standards such as the XML and URL (URI), and structures relationship between data and other resources. SPARQL is used for querying RDF formatted databases to discover relationships using semantic methods (Ježek and Mouček, 2015).

\section{Conclusion}

Wearable devices are gaining significant attention from many sectors including healthcare, sports, and entertainment. Both the implantable and the wearable devices are transforming traditional beliefs and work culture due to its features, such as remote monitoring and data collection. Wearables have the potential to change healthcare especially the quality of care for people that live in locations with little or no healthcare facilities. While wearable and implantable devices are important for quality healthcare, usable and quality data is required for machine-readable and artificial intelligence-driven clinical decisions. In this paper, several approaches to managing sensor data interoperability are discussed. Sensor data interoperability and various techniques for achieving sensor data interoperability were presented to enable users and potential users of healthcare data to understand how to make data usable. Specifically, state-of-the-art research on achieving sensor data interoperability is presented to enable healthcare providers, device manufacturers and policymakers to understand how to make sense of WBAN sensor data. There is a consensus among researchers that the patient has an important role play in data collection from wearable devices. As the push to reduce maternal death rate continues, there are challenges to ensure that WBAN sensor data is interoperable, shareable and available when the patient demands it. There are open challenges to sharing WBAN sensor data, such as the policies for sharing health data among healthcare providers within and across the border. As the use of wearables and implantable devices becomes popular, state governments and policymakers need to foster easy access to data for approved and certified new wearable devices that can be used in healthcare settings. Data privacy laws needs to be refined and institutionalized to protect users of wearable devices. Therefore, there is a need for creative and innovative models of sensor data interoperability. 


\section{References}

Ahmed, J. \& Ahmed, M., 2018. Semantic Web Approach of Integrating Big Data- A Review. International Journal of Computer Sciences and Engineering, 6(9), pp.529-532. Available at: doi:10.26438/ijcse/v6i9.529532.

Altar-Samuel, A., Costin, A. and Enache, D., 2015. RDF \& RDF QUERY LANGUAGESBUILDING BLOCKS FOR THE SEMANTIC WEB. Journal of Information Systems \& Operations Management, p.1.

Amoakoh-Coleman, M., Borgstein, A.B.J., Sondaal, S.F., Grobbee, D.E., Miltenburg, A.S., Verwijs, M., Ansah, E.K., Browne, J.L. and Klipstein-Grobusch, K., 2016. Effectiveness of mHealth interventions targeting health care workers to improve pregnancy outcomes in lowand middle-income countries: a systematic review. Journal of medical Internet research, 18(8), p.e226.

Azevedo, A.R.P., de Sousa, H.M.L., Monteiro, J.A.F. and Lima, A.R.N.P., 2015. Future perspectives of smartphone applications for rheumatic diseases self-management. Rheumatology international, 35(3), pp.419-431. Available at: doi:10.1007/s00296-014$3117-9$.

Bajaj, G., Agarwal, R., Singh, P., Georgantas, N. and Issarny, V., 2017. A study of existing Ontologies in the IoT-domain. arXiv preprint arXiv:1707.00112.

Beek, W., Schlobach, S. and van Harmelen, F., 2016, May. A contextualised semantics for owl: sameAs. In European Semantic Web Conference (pp. 405-419). Springer, Cham.

Bender, Duane, and Kamran Sartipi., 2013. "HL7 FHIR: An Agile and RESTful approach to healthcare information exchange." In Proceedings of the 26th IEEE international symposium on computer-based medical systems, pp. 326-331. IEEE.

Choi, J., Ha, M., Im, J., Byun, J., Kwon, K., Yoon, W., Kim, D., Heo, S. and Kim, D., 2015, April. The patient-centric mobile healthcare system enhancing sensor connectivity and data interoperability. In 2015 International Conference on Recent Advances in Internet of Things (RIoT) (pp. 1-6). IEEE.

Compton, M., Barnaghi, P., Bermudez, L., GarcíA-Castro, R., Corcho, O., Cox, S., Graybeal, J., Hauswirth, M., Henson, C., Herzog, A. and Huang, V., 2012. The SSN ontology of the $\mathrm{W} 3 \mathrm{C}$ semantic sensor network incubator group. Journal of Web Semantics, 17, pp.25-32.

Cornet, R., 2015. Combining archetypes with fast health interoperability resources in futureproof health information systems. Digital Healthcare Empowering Europeans: Proceedings of MIE2015, 210, p.180. Available at: doi:10.3233/978-1-61499-512-8-180.

de l'Aulnoit, A.H., Boudet, S., Génin, M., Gautier, P.F., Schiro, J., de l'Aulnoit, D.H. and Beuscart, R., 2018. Development of a smart mobile data module for fetal monitoring in EHealthcare. Journal of medical systems, 42(5), p.83.

Dimitrov, D.V., 2016. Medical internet of things and big data in healthcare. Healthcare informatics research, 22(3), pp.156-163.

Garde, S., Knaup, P., Hovenga, E.J. and Heard, S., 2007. Towards semantic interoperability for electronic health records. Methods of information in medicine, 46(03), pp.332-343. 
Joy Alatta / Wireless Body Area Network and Sensor Data Interoperability

Gay, V. and Leijdekkers, P., 2015. Bringing health and fitness data together for connected health care: mobile apps as enablers of interoperability. Journal of medical Internet research, 17(11), p.e260. Avaialable at: doi:10.2196/jmir.5094.

Hachem, S., Teixeira, T. and Issarny, V., 2011. Ontologies for the internet of things. In Proceedings of the 8th middleware doctoral symposium (pp. 1-6).

Haller, A., Janowicz, K., Cox, S.J., Lefrançois, M., Taylor, K., Le Phuoc, D., Lieberman, J., GarcíaCastro, R., Atkinson, R. and Stadler, C., 2019. The modular SSN ontology: A joint W3C and OGC standard specifying the semantics of sensors, observations, sampling, and actuation. Semantic Web, 10(1), pp.9-32.

He, Z., Tao, C., Bian, J., Dumontier, M. and Hogan, W.R., 2017. Semantics-powered healthcare engineering and data analytics. Journal of healthcare engineering, 2017.

Available at: doi:10.1155/2017/7983473.

Idrissou, A.K., Hoekstra, R., Van Harmelen, F., Khalili, A. and Van den Besselaar, P., 2017, December. Is my: sameAs the same as your: sameAs? Lenticular lenses for context-specific identity. In Proceedings of the Knowledge Capture Conference (pp. 1-8).

Honti, G.M. and Abonyi, J., 2019. A review of semantic sensor technologies in internet of things architectures. Complexity, 2019. Available at: doi:10.1155/2019/6473160.

Huang, C.Y. \& Chen, H.H., 2019. An Automatic Embedded Device Registration Procedure Based on the OGC SensorThings API. Sensors, 19(3), p.495. Available at: doi:10.3390/s19030495.

Huang, C.Y. and Wu, C.H., 2016. A web service protocol realizing interoperable internet of things tasking capability. Sensors, 16(9), p.1395. Available at: doi:10.3390/s16091395.

Hussain, A. and Wu, W., 2017. Sustainable Interoperability and Data Integration for the IoT-Based Information Systems. In 2017 IEEE International Conference on Internet of Things (iThings) and IEEE Green Computing and Communications (GreenCom) and IEEE Cyber, Physical and Social Computing (CPSCom) and IEEE Smart Data (SmartData) (pp. 824-829). IEEE.

Iroju, O., Soriyan, A., Gambo, I. and Olaleke, J., 2013. Interoperability in healthcare: benefits, challenges and resolutions. International Journal of Innovation and Applied Studies, 3(1), pp.262-270.

Ivaşcu, T., Frîncu, M. and Negru, V., 2016, August. Energy efficient sensors data stream model for real-time and continuous vital signs monitoring. In 2016 International Symposium on INnovations in Intelligent SysTems and Applications (INISTA) (pp. 1-5). IEEE.

Jabbar, S., Ullah, F., Khalid, S., Khan, M. and Han, K., 2017. Semantic interoperability in heterogeneous IoT infrastructure for healthcare. Wireless Communications and Mobile Computing, 2017.

Janowicz, K., Haller, A., Cox, S.J., Le Phuoc, D. and Lefrançois, M., 2019. SOSA: A lightweight ontology for sensors, observations, samples, and actuators. Journal of Web Semantics, 56, pp.1-10.

Ježek, P. and Mouček, R., 2015. Semantic framework for mapping object-oriented model to semantic web languages. Frontiers in neuroinformatics, 9, p.3. Available at: doi:10.3389/fninf.2015.00003.

Jirkovský, V., Obitko, M. and Mařík, V., 2016. Understanding data heterogeneity in the context of cyber-physical systems integration. IEEE Transactions on Industrial Informatics, 13(2), pp.660667.Available at: doi: 10.1109/RIOT.2015.7104911. 
Kaewkungwal, J., Singhasivanon, P., Khamsiriwatchara, A., Sawang, S., Meankaew, P. and Wechsart, A., 2010. Application of smart phone in" Better Border Healthcare Program": a module for mother and child care. BMC medical informatics and decision making, 10(1), p.69. Available at: doi:10.1186/1472-6947-10-69.

Kasthurirathne, S.N., Mamlin, B., Kumara, H., Grieve, G. and Biondich, P., 2015. Enabling better interoperability for healthcare: lessons in developing a standards based application programing interface for electronic medical record systems. Journal of medical systems, 39(11), p.182.

Kaur, A. et al., 2017. A Survey Paper on Wireless Body Area Network in Healthcare System. International Journal of Advanced Research in Computer Science and Software Engineering, 7(5), pp.708-712. Available at: doi:10.23956/ijarcsse/sv7i5/0306.

Kaur, N. \& Aggarwal, H., 2017. Evaluation of Information Retrieval Based Ontology Development Editors for Semantic Web. International Journal of Modern Education and Computer Science, 9(7), pp.63-73. Available at: doi:10.5815/ijmecs.2017.07.07.

Kim, H. \& Xie, B., 2015. Health literacy and internet- and mobile app-based health services: A systematic review of the literature. Proceedings of the Association for Information Science and Technology, 52(1), pp.1-4. Available at: doi:10.1002/pra2.2015.145052010075.

Kim, T.-Y. \& Kim, E.-J., 2016. Multi-hop WBAN configuration approach for wearable machine-tomachine systems. Multimedia Tools and Applications, 75(20), pp.12859-12878. Available at: doi: 10.1007/s11042-015-2832-x.

Kim, U.-H. et al., 2016. Analysis of Aggregation Delay for Multisource Sensor Data with On-Off Traffic Pattern in Wireless Body Area Networks. Sensors, 16(10), p.1622. Available at: doi:10.3390/s16101622.

Prashanth Kumar, K.N., Ravi Kumar, V. \& Raghuveer, K., 2017. A Survey on Semantic Web Technologies for the Internet of Things. 2017 International Conference on Current Trends in Computer, Electrical, Electronics and Communication (CTCEEC). Available at: doi:10.1109/ctceec.2017.8454974.

Kumar, R.B. et al., 2016. Automated integration of continuous glucose monitor data in the electronic health record using consumer technology. Journal of the American Medical Informatics Association, 23(3), pp.532-537. Available at: doi:10.1093/jamia/ocv206.

Lee, J.-H., 2016. Miniaturized Human Insertable Cardiac Monitoring System with Wireless Power Transmission Technique. Journal of Sensors, 2016, pp.1-7. Available at: doi:10.1155/2016/5374574.

Liyanage, H., Krause, P. \& De Lusignan, S., 2015. Using ontologies to improve semantic interoperability in health data. Journal of Innovation in Health Informatics, 22(2), pp.309-315. Available at: doi:10.14236/jhi.v22i2.159.

Lo, B.P.L., Ip, H. \& Yang, G.-Z., 2016. Transforming Health Care: Body Sensor Networks, Wearables, and the Internet of Things. IEEE Pulse, 7(1), pp.4-8. Available at: doi:10.1109/mpul.2015.2498474.

Maarala, A.I., Su, X. \& Riekki, J., 2017. Semantic Reasoning for Context-Aware Internet of Things Applications. IEEE Internet of Things Journal, 4(2), pp.461-473. Available at: doi:10.1109/jiot.2016.2587060. 
Joy Alatta / Wireless Body Area Network and Sensor Data Interoperability

Manda-Taylor, L., Sealy, D.A. and Roberts, J., 2017. Factors associated with delayed Antenatal Care attendance in Malawi: Results from a Qualitative study. Medical Journal of Zambia, 44(1), pp.17-25.

Mavrogiorgou, A. et al., 2019. IoT in Healthcare: Achieving Interoperability of High-Quality Data Acquired by IoT Medical Devices. Sensors, 19(9), p.1978. Available at: doi:10.3390/s19091978.

Mony, P.K. et al., 2018. Remote biomonitoring of temperatures in mothers and newborns: design, development and testing of a wearable sensor device in a tertiary-care hospital in southern India. BMJ Innovations, 4(2), pp.60-67. Available at: doi:10.1136/bmjinnov-2016-000153.

Nebeker, C., Linares-Orozco, R. and Crist, K., 2015. A Multi-Case Study of Research Using Mobile Imaging, Sensing and Tracking Technologies to Objectively Measure Behavior: Ethical Issues and Insights to Guide Responsible Research Practice. Journal of Research Administration, 46(1), pp.118137.

Noura, M., Atiquzzaman, M. \& Gaedke, M., 2018. Interoperability in Internet of Things: Taxonomies and Open Challenges. Mobile Networks and Applications, 24(3), pp.796-809. Available at: doi:10.1007/s11036-018-1089-9.

Peng, C., Goswami, P. \& Bai, G., 2018. Linking Health Web Services as Resource Graph by Semantic REST Resource Tagging. Procedia Computer Science, 141, pp.319-326. Available at: doi:10.1016/j.procs.2018.10.194.

Petit, A. \& Cambon, L., 2016. Exploratory study of the implications of research on the use of smart connected devices for prevention: a scoping review. BMC Public Health, 16(1). Available at: doi:10.1186/s12889-016-3225-4.

Prabhakaran, D., Ajay, V.S. \& Tandon, N., 2019. Strategic Opportunities for Leveraging Low-cost, High-impact Technological Innovations to Promote Cardiovascular Health in India. Ethnicity \& Disease, 29(Suppl 1), pp.145-152. Available at: doi:10.18865/ed.29.s1.145.

Puustjärvi, J. \& Puustjärvi, L., 2015. The Role of Smart Data in Smart Home: Health Monitoring Case. Procedia Computer Science, 69, pp.143-151. Available at: doi:10.1016/j.procs.2015.10.015.

Rahmani, Z. \& Brekke, M., 2013. Antenatal and obstetric care in Afghanistan - a qualitative study among health care receivers and health care providers. BMC Health Services Research, 13(1). Available at: doi:10.1186/1472-6963-13-166.

Reda, R., Piccinini, F. \& Carbonaro, A., 2018. Towards Consistent Data Representation in the IoT Healthcare Landscape. Proceedings of the 2018 International Conference on Digital Health - DH '18. Available at: doi:10.1145/3194658.3194668.

Reed, J.L. et al., 2015. E-health physical activity interventions and moderate-to-vigorous intensity physical activity levels among working-age women: a systematic review protocol. Systematic Reviews, 4(1). Available at: doi:10.1186/2046-4053-4-3.

Rhayem, A., Mhiri, M.B.A. \& Gargouri, F., 2017. HealthIoT Ontology for Data Semantic Representation and Interpretation Obtained from Medical Connected Objects. 2017 IEEE/ACS 14th International Conference on Computer Systems and Applications (AICCSA). Available at: doi:10.1109/aiccsa.2017.171.

Roy, S.N., Srivastava, S.K. and Gururajan, R., 2018. Integrating Wearable Devices and Recommendation System: Towards a Next Generation Healthcare Service Delivery. Journal of Information Technology Theory and Application (JITTA), 19(4), p.2. 
Saripalle, R.K., 2019. Fast Health Interoperability Resources (FHIR). International Journal of EHealth and Medical Communications, 10(1), pp.76-93. Available at: doi:10.4018/ijehmc.2019010105.

Say, L. et al., 2014. Global causes of maternal death: a WHO systematic analysis. The Lancet Global Health, 2(6), pp.e323-e333. Available at: doi:10.1016/s2214-109x(14)70227-x.

Seydoux, N. et al., 2016. IoT-O, a Core-Domain IoT Ontology to Represent Connected Devices Networks. Knowledge Engineering and Knowledge Management, pp.561-576. Available at: doi:10.1007/978-3-319-49004-5_36.

Shokeen, S. \& Parkash, D., 2019. A Systematic Review of Wireless Body Area Network. 2019 International Conference on Automation, Computational and Technology Management (ICACTM). Available at: doi:10.1109/icactm.2019.8776847.

Saripalle, R.K., 2019. Fast Health Interoperability Resources (FHIR): Current status in the healthcare system. International Journal of E-Health and Medical Communications (IJEHMC), 10(1), pp.76-93.

Studeny, J. and Coustasse, A., 2014. Personal health records: is rapid adoption hindering interoperability?. Perspectives in Health Information Management, 11(Summer).

Sudjai, M., Safaei, F., Wysocki, T. and Phung, S.L., 2016. High-speed adaptive wireless body area networks. EURASIP Journal on Wireless Communications and Networking, 2016(1), pp.1-16. Available at: doi:10.1186/s13638-016-0772-4.

Vengadeswaran, A., Neuhaus, P., Hegselmann, S., Storf, H. and Kadioglu, D., 2019. Semantically Annotated Metadata: Interconnecting Samply. MDR and MDM-Portal. Studies in health technology and informatics, 267, pp.86-92.

WHO (2019). Maternal Mortality. Available at: $<$ https://www.who.int/news-room/factsheets/detail/maternal-mortality $>$. [Accessed December 07, 2019].

W3C. Semantic Web. Available at: https://www.w3.org/standards/semanticweb/. [Accessed: December 12, 2019.

Yilmaz, T., Foster, R. \& Hao, Y., 2010. Detecting Vital Signs with Wearable Wireless Sensors. Sensors, 10(12), pp.10837-10862. Available at: doi:10.3390/s101210837.

Zhang, P. et al., 2018. Blockchain Technology Use Cases in Healthcare. Blockchain Technology: Platforms, Tools and Use Cases, pp.1-41. Available at: doi:10.1016/bs.adcom.2018.03.006. 